\title{
Hysteresis of Ionization Waves
}

\author{
A. Dinklage \\ Max-Planck-Institut für Plasmaphysik, EURATOM-Association, \\ Wendelsteinstr. 1, 17491 Greifswald, Germany* \\ B. Bruhn, H. Testrich \\ Institut für Physik, E.-M.-Arndt Universität Greifswald, \\ Felix-Hausdorff-Str. 6, 17487 Greifswald, Germany \\ C. Wilke \\ Institut für Niedertemperaturplasmaphysik, \\ Felix-Hausdorff-Str. 2, \\ 17489 Greifswald, Germany \\ April 4, 2008
}

\begin{abstract}
A quasi-logistic, non-linear model for ionization wave modes is introduced. Modes are due to finite size of the discharge and current feed-back. The model consists of competing coupled modes and it incorporates spatial wave amplitude saturation. The hysteresis of wave mode transitions under current variation is reproduced. Side-bands are predicted by the model and found in experimental data. The ad-hoc model is equivalent to a general - so-called universal - approach from bifurcation theory.

PACS numbers: 52.35.Py,52.80.Hc,05.45.+b
\end{abstract}

*Electronic address: dinklage@ipp.mpg.de 


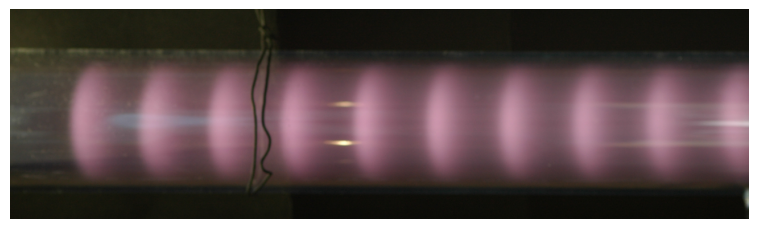

FIG. 1: Photograph of standing ionization waves in an $\mathrm{O}_{2}$ dc glow discharge. The luminous layers are seen through a glass cylinder confining the discharge plasma.

\section{INTRODUCTION}

Ionization waves are a result of an ubiquitous instability in low temperature plasmas. The waves manifest as structured regular layers representing variations of the plasma density, densities of excited state atoms and the axial electric field as well as global modulations of the discharge current. The fluctuations particularly affect the light emission as shown in Fig.1. Due to their spectacular visibility, ionization waves might be the oldest experimentally documented non-linear model system [1]. The multifaceted physics aspects and its experimental accessibility make ionization waves in discharges a textbook example for low-temperature plasma physics as well as for non-linear dynamics.

Control parameters of the experiment are the neutral gas pressure $p$, the discharge length $L$ and the discharge driven current $I$. The current is the superior control parameter for an exact and flexible manipulation of the system. It is even possible to superimpose current noise at different amplitudes to perform a variety of studies relevant to topical aspects of non-linear physics, e.g. the behavior of saturated waves with superposed artificial noise [2] which is accessible in discharges [3]. Furthermore, there is a variety of investigations addressing different topics of non-linear behavior in these plasmas, e.g. [4-7].

Although the phenomenon of structured discharges is long known, important aspects of ionization waves could be revealed quite recently only, particulary kinetic mechanisms (see e.g. [8]) and dynamic properties [9]. As usual for non-linear phenomena, the different views recover different aspects of the dynamics. However, the approaches limited are in many cases or may become too complicated to be understandable at a glance. In the case of ionization waves, a full kinetic description fails to explain the dynamics of saturated wave modes and - on the other hand - a description of macroscopic dynamics by bifurcation theory does not self-consistently recover the interplay of electron kinetics and wave properties. Therefore, 
simplified models of the dynamics are required to link the different aspects focusing on the leading physical effects generating the observed dynamics.

The macroscopic dynamics of the waves is relevant to applications, e.g. in order to control the discharges for lighting purposes and materials processing [10]. Consequently, models for the macroscopic behavior are valuable for design considerations in discharge applications. For basic non-linear dynamics, such models may be employed to test different descriptive approaches.

The specific macroscopic dynamic phenomenon addressed in this paper is the hysteresis of ionization wave modes of the so called p-type [15]. The wave modes develop due to the finite length of the discharge and feed-back of the discharge current oscillations [11]. Hysteresis means in this context that multiple wave mode states for a given control parameter exist, but only one state is selected depending on the history of the control parameters or - in other words - how the system was prepared. For the present case the options for system preparation are the increase or decrease of the discharge current.

The specific phenomenology of the wave modes and the hysteresis will be introduced next. The main statements of the paper follow then: first, a quasi-logistic model will be motivated and second the model will be validated theoretically by means of a universal description from bifurcation theory. The results are discussed in the end of this paper.

\section{PHENOMENOLOGY OF IONIZATION WAVE MODES IN DC GLOW DIS- CHARGES}

This section introduces the phenomenology of ionization wave modes and specifies the hysteresis phenomenon in more detail. Concise reviews introducing ionization waves and their dynamical aspects may be found in $[12,13]$.

The discharge is a de glow discharge operated at low currents and low pressures, typical values are shown Fig. 2. In this paper, the sealed cylindrical discharge tube is filled with neon gas. A set-up is shown in Fig. 3. The dynamics due to ionization waves are observed by optical detectors allowing a non-invasive examination of the sensitive space-time dynamics. Please note, that detailed parameters of the discharges presented in this paper may vary in pressure and radius according to the figure captions. These differences in parameters may lead to differences in the occurrence of the wave modes [14]. 


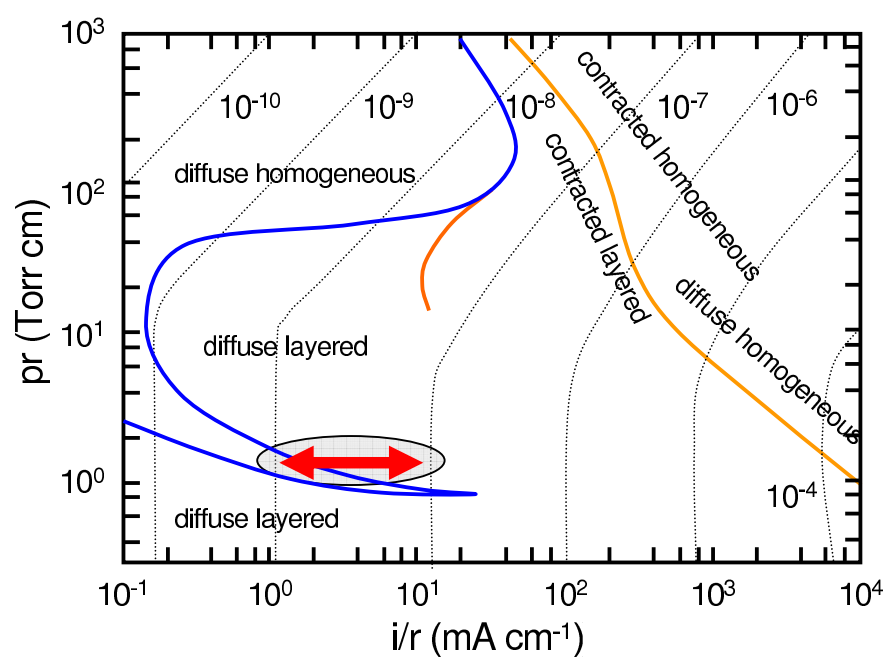

FIG. 2: Occurrence of ionization waves in neon discharge in figures of the similarity parameters $i / r$ ( $i$ : discharge current, $r$ : radius of the tube) and $\operatorname{pr}$ ( $p$ : neutral gas pressure). The regions indicate the occurrence of different discharge modes, the shaded region indicates typical parameters as used in this paper. The arrow indicates the principal variation of control parameters performed here (adapted from [15]).

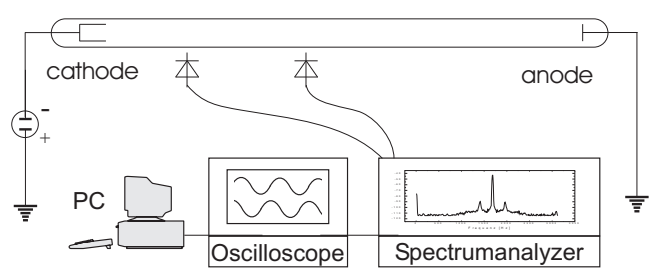

FIG. 3: Schematic diagram of the experimental set-up for the wave dynamics investigation. Linear arrays of photo-sensors allow a full detection of the wave patterns.).

For typical discharges investigated here, Fig. 2 shows a transition of the discharge mode from diffuse-homogeneous to diffuse-layered when the discharge current is varied for constant pressure. This transition is due to the occurrence of the ionization instability [13]. In the terminology of non-linear dynamics, this is a supercritical Hopf-bifuraction for p-waves in the considered parameter range. The observed low amplitude fluctuations may be regarded as noise sustained structures $[16,17]$.

The occurrence of the wave modes modes depends on the discharge length and the feedback of the discharge current [11]. Fig. 4 documents the effect of the discharge length. For this purpose, a discharge with variable anode position was prepared allowing a variation of the discharge length. The sequence of plots shows the power spectral density of light 


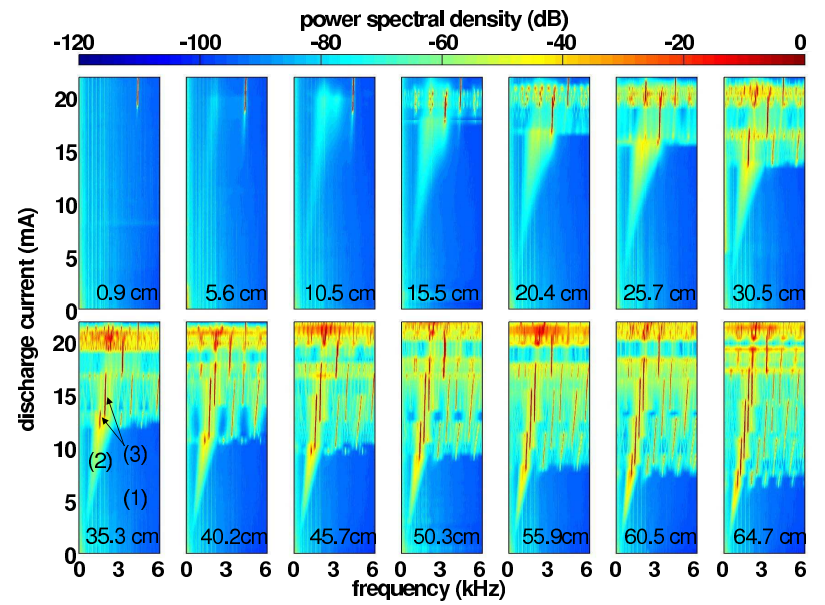

FIG. 4: Spectra (power spectral density, PSD) of light fluctuations $0.4 \mathrm{~cm}$ in front to the anode of a dc glow discharge in neon $(p=1.5$ Torr, $r=1 \mathrm{~cm})$ vs. discharge current and frequency. The plots refer to cathode-anode distances as indicated. The labeling of qualitatively different PSD (left plot in the bottom row) (1) - (3) refers to damped ionization instability (1), incoherent ionization instability (2) and ionization wave modes (3).

fluctuations close to the anode of the discharge where the fluctuations become maximum. The power spectral density was recorded by a spectrum analyzer fed by a photo diode. The figure is an overlay of spectra taken at discharge currents separated by $\delta i=0.1 \mathrm{~mA}$. For the later discussion of wave modes, it is important to note that the overlay is produced by a sequence of increasing and decreasing currents. Qualitatively, three power spectral density levels can be identified in Fig. 4: (1) a 1/f noise region which corresponds indicates vanishing ionization instability, (2) an intermediate fluctuation level which indicates an amplified ionization instability (i.e. its growth rate is positive $\gamma>0$ ) and (3) sharp lines indicate the occurrence of ionization wave modes.

These wave modes can be characterized by a spatial mode number $n$ according to $n=L / \lambda$ where $\lambda$ is the wavelength. It is a peculiarity of ionization waves that group and phase velocity are directed oppositely due to the dispersion of ionization waves $(f \sim \lambda, f$ is the wave frequency). The group velocity points from cathode to anode of the discharge.

Two aspects relevant to the following discussion can be concluded from Fig. 4. First, ionization wave modes (level (3) in Fig. 4) occur at higher discharge currents than the ionization instability. Differently to the instability (level (2) in Fig. 4) the power spectral density rises in its amplitude non-continuously when the current is increased. This indicates 


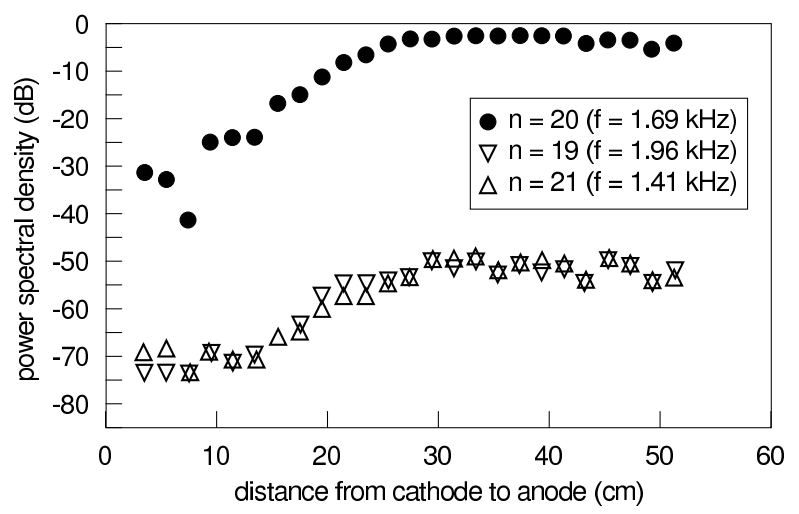

FIG. 5: Spatial distribution of harmonic light oscillations (neon discharge, length $54 \mathrm{~cm}$, discharge current $9.54 \mathrm{~mA}$, neutral gas pressure 1.6 Torr, radius $1.1 \mathrm{~cm}$ ).

a sub-critical Hopf bifurcation. The observed sequence of the ionization instability to modes shows that a certain minimum discharge length (spacing between cathode and anode) is required for the existence of modes. The threshold of modes is the smaller the longer the discharge becomes. This is a result of the feed-back mechanism. To support this conclusion, Fig. 5 shows the distribution of the light fluctuation power of a different wave modes (at fixed frequency). The wave amplitude rises exponentially from the cathode region towards the anode saturating at a finite distance from the cathode. This amplitude dependence is due to the propagation direction of ionization waves in neon (group velocity from cathode to anode). If a sufficiently high level of fluctuations arrives at the anode, modes develop due to finite length and current feed-back in the external circuit of the discharge. The feedback also fixes the phases of the fluctuations both in the discharge and the external driving circuit.

Fig. 5 also indicates strongly suppressed side-bands. These measurements were taken due to the model predictions described later. Side-bands were also identified to play an important role in the transition process $[18,19]$ : It was experimentally proven that the mode transition is a non-linear process in which a long-wavelength instability (Eckhaus instability) provides an energy transfer from the initial to the final wave mode. This process may be described as a spatio-temporal periodic pulling process. The present observation explains how the instability is triggered: the strongly suppressed neighboring modes permanently exist and grow when a mode transition takes place.

A more detailed view of the stability of wave modes is shown in Fig. 6. The diagram reflects the governing wave mode if the discharge current is varied. Starting from small 


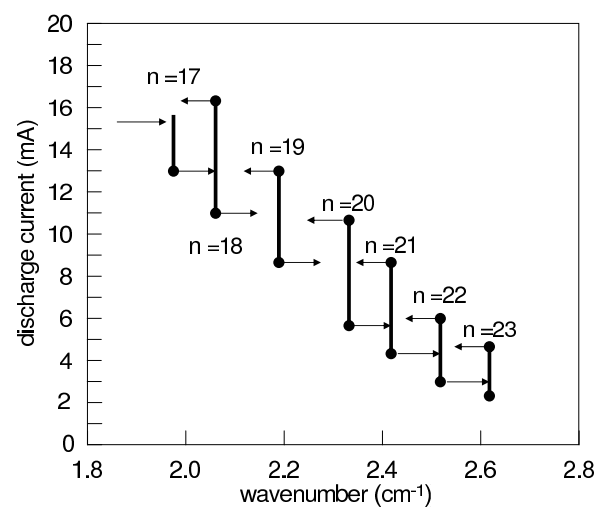

FIG. 6: Experimental stability diagram of ionization wave modes. $n$ is the longitudinal wave mode number from wavelength measurements (neon discharge, length $54 \mathrm{~cm}$, discharge current $9.54 \mathrm{~mA}$, neutral gas pressure 1.6 Torr, radius $1.1 \mathrm{~cm})$.

currents, Fig. 6 shows the onset of modes $(n=23)$. Increasing the current leads to mode transitions ultimately leading after a sequence of $\Delta n=-1$ transitions to mode number $n=17$. Decreasing the current increase the mode number $(\Delta n=+1)$ but the transition current is lower than the current for the inverse transition. Ramping the current up and down exhibits the hysteresis addressed here.

\section{A QUASI-LOGISTIC MODEL}

\section{A. Motivation}

First, the reasoning leading to the ad-hoc model describing the mode transition phenomenology is given.

The generic model for a wave-wave interaction is a third order non-linear equation (see Eq. 4 and [23]). From a physical point of view, the nonlinearity can be associated to wave intensities $\left(\sim A_{i}^{2}\right)$ fed by the free energy (represented by an energy density $E$ ) in the discharge.

Then, the saturation of the wave amplitudes is considered by the free energy parameter $E$ resulting in a limitation of the wave amplitude. This also reflects the fact that discharges are open, driven systems far from equilibrium. Since the plasma is driven by electric energy transferred to the electrons, $E$ is limited by the discharge current and the maximum voltage 
applied between cathode and anode. The free energy parameter is a function of applied electric power or can be rephrased as a function of discharge current. It is an experimental observation that the saturated wave amplitude does not change after mode transition at constant discharge currents. This also justifies the introduction of a free energy which is shared by all excitable waves.

As supported by Fig. 4, the frequency range of positive spatial amplification of the ionization instability $\gamma>0$ is quite broad if the discharge current is not too small. Particulary, several mode numbers can be excited which is a prerequisite for the observed occurrence of different predominant wave modes at a given discharge current and the observed hysteresis.

The model is termed logistic because it reflects the idea that different wave modes with $\gamma>0$ compete for the free energy. The mode competition is ad-hoc introduced by a mode coupling factor $\kappa$ which allows a transfer of energy between co-existing modes.

A non-explicitly time dependent, stationary spatial model is considered because the wave amplitude was found to be stationary in a finite time after mode transitions. The basic part of the model reflects the spatial amplification $\gamma$ as well as the saturation of the wave modes as shown in Fig. 5.

Summarizing the introduced picture one attains the following model:

$$
\frac{d A_{i}}{d z}=\frac{A_{i}}{E} \gamma_{i} \times\left(E-A_{i}^{2}-\kappa \times \sum_{i \neq k} A_{k}^{2}\right)
$$

which is finally called quasi-logistic since it resembles the logistic model except the saturation is given by a cubic rather than a quadratic term. The notation in Eq. 1 is $A_{i}$ to be the amplitude of the $i^{t h}$ wave mode, $z$ is the distance from the cathode, $\gamma_{i}$ the mode amplification, $E$ the plasma's free energy and $\kappa$ the coupling parameter for different wave modes. $\kappa>1$ can be interpreted to account for power losses since the free energy $E$ and the wave energies do not compensate each other. Power losses occur due to thermal convection or radiation in the discharge.

For the analysis of the model in Eq. 1, the current feed-back in the external circuit enters twofold. First, as mentioned previously it fixes the waves' phases at the cathode and the anode resulting in discrete wave modes $i$. Second, the feed-back introduces a constraint for the boundary condition to be obeyed when Eq. 1 is solved. The ratio of the amplitude of a wave mode $A_{i}$ at the cathode $(z=0)$ to its amplitude at the anode $(z=L$ where $L$ is the 


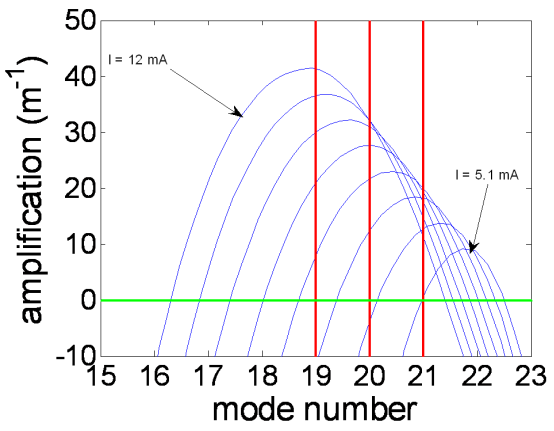

FIG. 7: Spatial amplifications for ionization wave modes as a function of wavenumber at different discharge currents.

length of the discharge) is the feed-back factor $r$ :

$$
r=\frac{A_{i}(z=0)}{A_{i}(z=L)}
$$

Furthermore, thermal fluctuations introduce a noise level $\xi$ (see also Fig. 4) which seed the excitable system. The boundary condition for Eq.1 is therefore given by

$$
A_{i}(z=0)=\max \left(r_{i} \times A_{i}(z=l), \xi\right)
$$

The model is solved iteratively for a coupled system of differential equations 1. Practically, only modes with $\gamma_{i}>0$ are considered.

\section{B. Derivation of Model Parameters from Experimental Results}

For the purpose of clarity we focus on a situation for which three wave modes compete. This corresponds to the measurement shown in Fig. 5. The model parameters in Eq. 1 can be derived from experimental results. The spatial amplification can be derived from mesurements, see Fig. 5. Since the dispersion of ionization waves $f=f(\lambda)$ is accessible as well, the wave numbers can be determined and the amplification $\gamma_{i}$ can be expressed as a function of wave-number $i$ which is shown in Fig. 7 .

As Fig. 7 indicates, the spatial amplification is well approximated by a unimodal function in the considered parameter range. The maximum spatial amplification becomes positive above a critical current (supercritical Hopf bifurcation). Increasing the discharge current 
leads to an increased maximum and a shift of the maximum to smaller wave numbers corresponding to higher frequencies.

It is instructive to consider the spatial amplifications for fixed wave numbers under current variations by Fig. 7. First, it is evident that currents exist for which the three wave modes considered have a positive amplification. For a given wave mode an increase of the the current results in a minimum and maximum current for which $\gamma_{i}>0$. This is the current region for which a self-excited wave can exist. In between the limiting currents, a current for maximum amplification exists.

If two adjacent wave modes are compared, both the $\gamma_{i}>0$ current range and the maximum amplification are shifted. Hence, for a given current there is exactly one wave mode with a maximum amplification. If the amplification would be the leading parameter for the selection of a wave mode only, a unique wave mode should be attained if a current is chosen. Particularly, no hysteresis phenomena would occur.

But hysteresis may occur if the feed-back boundary conditions (cf. Eq.6) are considered. The feed-back factor $r$ is derived from Fig.5 as well as the typical noise level normalized to the saturated wave amplitude at the anode $(z=L)$. For the experimental case modeled here, the values were $r=1 / 141.3$ and $\xi / E=2 \times 10^{-5}$.

\section{Model Results}

Employing the model parameters derived in the previous section, there is one parameter left not directly accessible. This is the coupling parameter $\kappa$ which was varied in the modeling. A value of $\kappa=1.2$ was found to fit with the experimental stability diagram best. $\kappa$ determines the extent of the hysteresis region.

The modeled stability diagram for the modes from Fig. 5 is shown in Fig. 8. For the calculation of the stability diagram, the set of coupled differential equations was solved with the boundary condition in Eq. 3. The model equations were solved iteratively, i.e. after solving the model with the initial boundary conditions, new boundary conditions were derived according Eq. 3 to account for the feed-back. If the fed-back amplitude at the anode $(z=L)$ is below the noise level, the wave amplitude cannot rise in the iterative procedure. The iteration was terminated after iteration $k$ when the overall solution for the amplitude does not vary more than $\left(A^{(k-1)}(z)-A^{(k)}(z)\right) / A^{(k}(z)<10^{-6}$. 


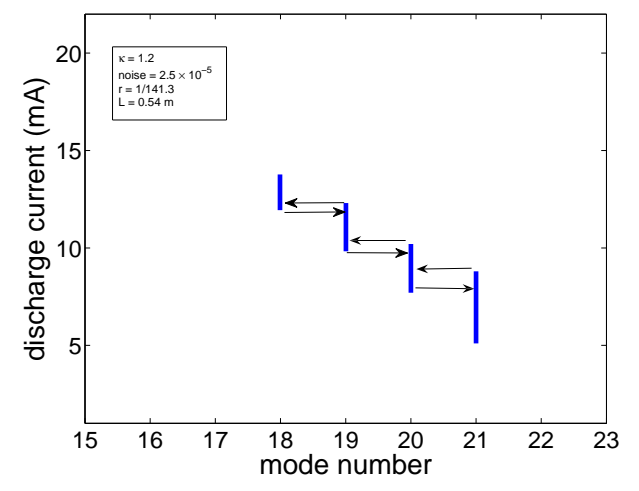

FIG. 8: Modeled stability diagram.

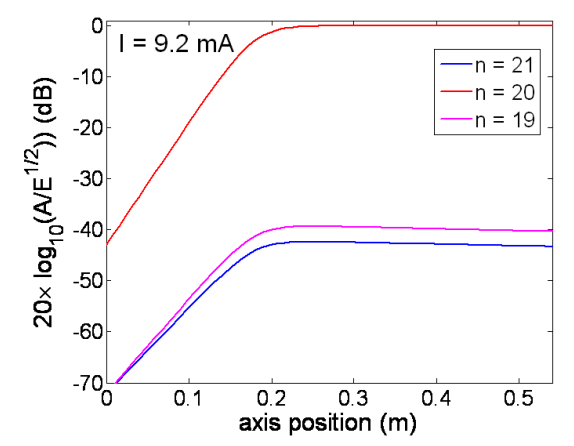

FIG. 9: Spatial distribution wave amplitudes.

A typical spatial variation of the stationary solution of the wave amplitudes is shown in Fig.9.

It is an outcome of the model that modes $\gamma>0$ exist but at a small amplitude. Again, this prediction was experimentally confirmed (Fig. 5).

The stability investigation started at low currents where all modes considered were damped. At a threshold of about $I=4.8 \mathrm{~mA}$ mode $n=21$ occurs since $\gamma_{n=21}>0$. Now the discharge current is raised in small steps $\Delta I=0.01 \mathrm{~mA}$ and the initial boundary conditions was taken as the stationary solution from the previous step. This resembles the experimental procedure exactly. Along the increase of discharge currents mode jumps occurs to $n=20$ and $n=21$ occur respectively. Decreasing the current leads to opposite jumps but at a lower current. 
Fig. 8 shows that the experimental result can be modeled. The explanation why a wave mode $j$ governs the dynamics of a discharge even in cases were a more unstable mode $i$ exists, i.e. $\gamma_{j}<\gamma_{i}$, is given by the feed-back mechanism. If the discharge is prepared in a state $k$, the feed-back amplitude is larger than the noise excited amplitude of a mode $q$ $A_{k}(z=0)>A_{q}(z=0)$ since $r \times A_{q}(z=L)>\xi$. Mode transition occur if the feed back amplitude of mode $k$ gets smaller than the feed-back amplitude of mode $q$. Therefore, the mode $k$ remains the governing wave mode even though its linear spatial amplification is even smaller than that of mode $q$. The transition criterion is hardly expressed in an analytic form since the model parameters enter in the non-linear solution of the coupled differential equations. Moreover, the iterative mapping to mimic the feed-back makes a closed analytic expression for the transition condition impossible. Nonetheless, the basic mechanism could be comprehended and the model is validated by its prediction of strongly suppressed sid-bands.

\section{LINK TO UNIVERSAL DESCRIPTIONS}

The theoretical explanation of the stability diagram of ionization waves may be attempted in the framework of a hydrodynamic description of the plasma. A numerical analysis of the underlying set of balance equations (c.f. ref.[20]) provides the asymmetric stability band observed in neon, which is the prerequisite for the understanding of the hysteresis phenomenon. Moreover, a universal description exists in terms of an amplitude equation of higher order which is valid in the weakly nonlinear region [21]. These methods give information on the global properties of the stability diagram, i.e. the asymmetric shape of the band that is bent to smaller wave numbers. But the details of the mode transitions which are connected with the interaction of neighboring modes can not be obtained in this way. These properties require a local model of the mode-mode interactions.

Fortunately, there exists such an universal description in terms of a codimension-two bifurcation of the Hopf-Hopf type [22]. Note that a mode transition can also be initiated by variation of the length of the positive column [11]. Figure 10 shows a sketch of the marginal curves near the onset of instability of two neighboring modes. I denotes the discharge current and $k$ is the wave number. The stability boundaries $\mu_{1}=0, \mu_{2}=0$ must be calculated from the dispersion relation of the underlying plasma model [22]. 


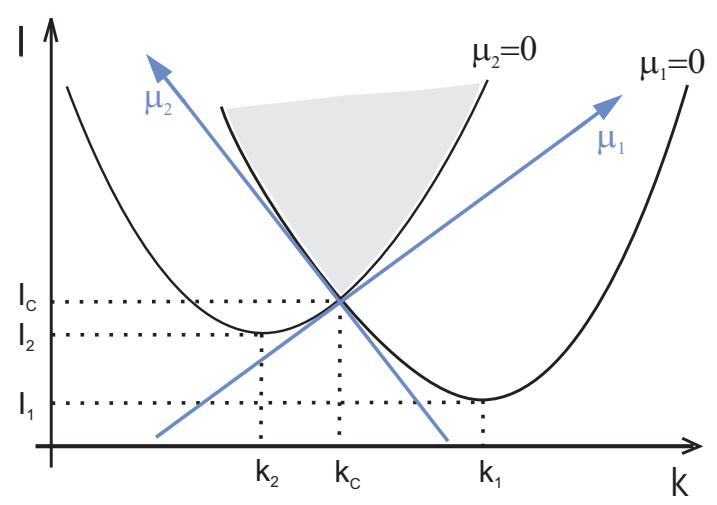

FIG. 10: Sketch of the instability curves of two neighboring wave modes. The grey sector indicates the coexistence region of both modes where the transition occurs.

Below these curves the homogeneous state is stable. Crossing a single marginal mode curve by increasing the current, for example at $I=I_{1}$, a Hopf bifurcation takes place and above this critical value the mode has a well defined amplitude. Here we have chosen $I_{1}<I_{2}$ according to the experimental data in a neon plasma. In ref. [22] all modes have the same critical current which is a consequence of the used periodic boundary conditions. At the critical point $I_{c}, k_{c}$ a Hopf-Hopf bifurcation can be observed and above this point there is a region where both modes coexist. The dynamics near the critical point $I_{c}, k_{c}$ can be described by the normal form equations up to third order in nonlinearity:

$$
\begin{aligned}
& \dot{r}_{1}=\mu_{1} r_{1}+\left(a_{1} r_{1}^{2}+b_{1} r_{2}^{2}\right) r_{1}+\ldots \\
& \dot{r}_{2}=\mu_{2} r_{2}+\left(a_{2} r_{2}^{2}+b_{2} r_{1}^{2}\right) r_{2}+\ldots
\end{aligned}
$$

More precisely, these are the truncated equations (c.f. Ref.[23]) of the normal form which takes into account the real wave amplitudes $r_{1}, r_{2}$ only. $\mu_{1,2}=\mu_{1,2}(I, k)$ are denoted as the unfolding parameters. A power series expansion near the critical point up to the first order in $I, k$ yields

$$
\begin{aligned}
& \mu_{1}(I, k)=\alpha\left(I-I_{c}+\frac{\left(I_{1}-I_{c}\right)}{\left(k_{c}-k_{1}\right)}\left(k-k_{c}\right)\right) \\
& \mu_{2}(I, k)=\beta\left(I-I_{c}+\frac{\left(I_{2}-I_{c}\right)}{\left(k_{c}-k_{2}\right)}\left(k-k_{c}\right)\right),
\end{aligned}
$$

where $\alpha$ and $\beta$ are positive constants that can be determined by means of the tangents at the critical point (c.f. Fig.10). Eq. (5) connects the unfolding parameters to the physical 
quantities current $I$ and wave number $k$.

Since we deal with wave motions, i.e. $\frac{d}{d t} \rightarrow c \frac{d}{d z}$, where $c$ is the group velocity of ionization waves, there is a one to one relation to the quasi-logistic model discussed in section III. We find the relation between the coefficients:

$$
\mu_{i} \rightarrow \gamma_{i}, \quad a_{1}=a_{2} \rightarrow \frac{-1}{E}, \quad b_{1}=b_{2} \rightarrow \frac{-\kappa}{E}
$$

Then the behavior of the quasi-logistic model can be interpreted in terms of the stationary solutions of Eq. (4). For our purpose the most important asymptotic solution is the coexisting state with $r_{1,2} \neq 0$ that can be written as

$$
r_{1}=\sqrt{\frac{b_{1} \mu_{2}-a_{2} \mu_{1}}{a_{1} a_{2}-b_{1} b_{2}}}, r_{2}=\sqrt{\frac{b_{2} \mu_{1}-a_{1} \mu_{2}}{a_{1} a_{2}-b_{1} b_{2}}} .
$$

Using the parameters

$$
\delta=\frac{b_{2}}{a_{1}}, \quad \theta=\frac{b_{1}}{a_{2}}
$$

and the constraints

$$
\delta>0, \quad \theta>0, \quad \delta \theta>1,
$$

the coexisting state of two modes exist in a range $\delta \mu_{1}>\mu_{2}>\mu_{1} / \theta$. A stability analysis shows that the fixed point (7) is hyperbolic with a 1-dimensional stable and a 1-dimensional unstable manifold, i.e. the coexisting state of both modes is unstable. This corresponds to the gray sector in Fig. 10. For experimental situations, a transient phase of unstable coexistence of both modes finally leads to a stable fixed point, i.e. a regular wave motion. The transition mechanism will be discussed in the following.

Figure 11 shows the corresponding bifurcation diagram. Note that [22] contains misprints for this case. In the phenomenological model the nonlinear coupling parameter $\kappa$ with a value somewhat larger than 1 is considered. This case corresponds to $b_{1} b_{2}-a_{1} a_{2}=\varepsilon>0$ with $\varepsilon<<1$ for the normal form equation. Thus, the two eigenvalues $\lambda_{+,-}$of the stability problem have a different magnitude, given by

$$
\lambda_{+}=O(\varepsilon), \quad \lambda_{-}=O(1)
$$

where $\lambda_{+}$denotes the positive eigenvalue. We shall denote this situation as a weakly unstable fixed point. For any initial condition the system (4) provides a well defined final state $r_{1} \neq 0, r_{2}=0$ or $r_{1}=0, r_{2} \neq 0$ in the limit of an infinite time evolution $t \rightarrow \infty$ or in 


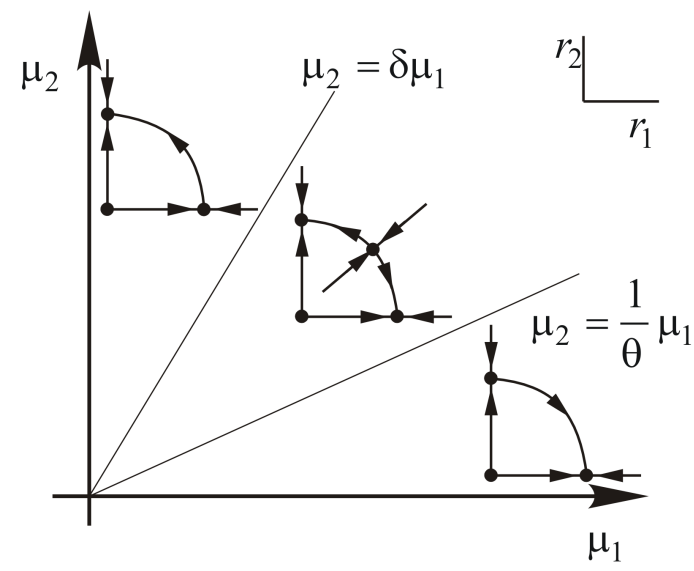

FIG. 11: Bifurcation diagram for the coexistence region in the case: $\delta \theta>1, \mu_{1,2}>0$.

the limit of an infinite long system. The boundary of attraction of the single modes is a heteroclinic orbit that connects the trivial solution $r_{1}=r_{2}=0$ with the fixed point defined by (7). Note, that this orbit depends on the actual values of $k$ and $I$. A mode transition occurs if there is a crossing of the hetroclinic orbit.

In a system of finite length $L$ combined with an iterative feed-back, a more complicated situation arises. We discuss the mode transition in Fig.12 which represents a sketch of the dynamical states within the amplitude space. We chose parameters $k=k_{1}, \mu_{i}=\mu_{i}\left(k_{1}, I\right)$ and initial conditions which realize mode 1 and mode 2 has the typical noise level. A first step of the iteration provides new initial data that are located near the origin $r_{1}=r_{2}=0$ if the feed-back parameter $r$ is sufficiently small. Of course, the new initial data must be larger than the noise level. The following finite time step results in amplitudes $r_{i} \neq 0$ which are in a neighborhood of the solution (7) because this point has a strongly attractive and a weakly repulsive direction, respectively. Further iterations provide the stable finite amplitudes $r_{i} \neq 0$ but with $r_{1}>r_{2}$. The final ratio $r_{2} / r_{1}$ depends upon the coupling as well as on the feed-back. By increasing the current $I$ one changes the position of the fixed point $(7)$ within the $\left(r_{1}, r_{2}\right)$-plane and therefore one changes the position of the heteroclinic orbit which is the basin boundary between both modes. At a critical current the feed-back sets initial conditions that cross the basin boundary and the mode transition occurs. A similar picture can be obtained if the starting state is mode 2 with $k=k_{2}$ and the current is decreased. But now the critical current at which the transition occurs is different from the first case by an increasing current. Of course, this is a consequence of the dependence of 


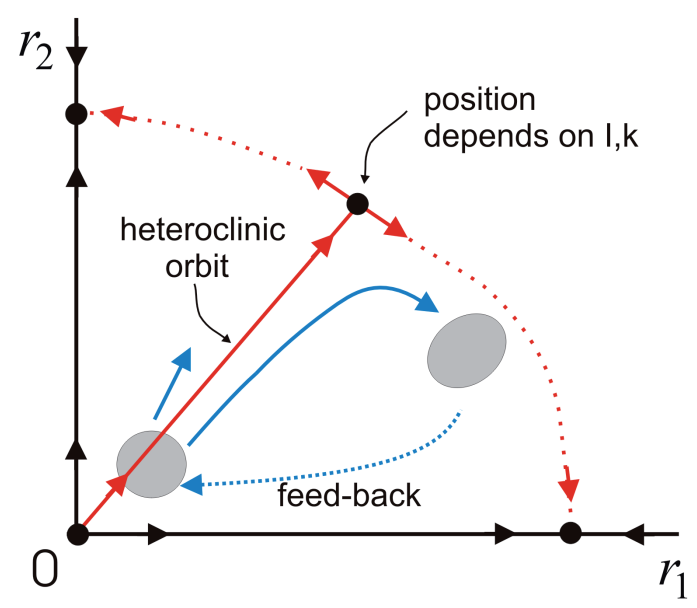

FIG. 12: Sketch of the dynamical states and the basin boundaries for a system of finite length.

the fixed point (7) and of the associated heterocyclic orbit upon the parameter $k_{2}$ that has a different value from $k_{1}$. Using this explanation of the hysteresis, we conclude that there must be at least a weak dependence of the transition current upon the initial conditions.

\section{CONCLUSION}

A quasi-logistic model was introduced. The model resembles a wave mode competition under consideration of spatial growth rate, amplitude saturation, energy dissipation, finite system length and feed-back. The feed-back mechanism is identified to be the reason for stabilizing a wave mode even if its growth rate is less than the growth rate of another unstable mode. Consequently, the system may stay in its original mode under small variations of control parameters. However, if the variation is too large even the feed-back cannot compensate the enhanced growth of the most unstable mode leading to a mode transition. In other words, the preparation of the discharge may determine which ionization wave mode governs. This is the observed hysteresis.

Basically, the universal approach accounts for the pre-history of the discharge as well by the control parameter dependencies of the fixed points representing the wave solutions. This explains the hysteresis in the bifurcation model as well.

It is interesting to note, that noise is an important ingredient in both approaches since it seeds all unstable wave modes. This is important to allow the discharge to select between 
the modes and it was shown in this paper that these wave modes exist as strongly suppressed side-bands.

The main outcome of this paper is that the quasi-logistic model motivated by general considerations of wave-wave bifurcations completes the theoretical picture of the discharge. The description of the hysteresis in terms of a mode competition links the universal description with dynamics aspects of the mode transitions. Particulary, aspects of the formerly observed spatio-temporal dynamics (Eckhaus instability, spatio-temporal periodic pulling) are linked to the universal description by the hysteresis model presented in this study.

\section{Acknowledgements}

This work was conducted within Sonderforschungsbereich TR24 (projects B1 and B8) funded by Deutsche Forschungsgemeinschaft.

[1] M. Abria, Annales des Chimie et de Physique $3^{e}$ serie 7, 462 (1843).

[2] O.V. Ushakov, H.J. Wunsche, F. Henneberger, I.A. Kohovanov, L. Schimansky-Geier, M.A. Zaks, Phys. Rev. Lett. 95, 123903 (2005).

[3] A. Dinklage, C. Wilke, T. Klinger, Phys. Plasmas 6, 2968 (1999).

[4] R. Kozakov, C. Wilke, B. Bruhn, Phys. Lett. A 360, 448 (2007).

[5] I. Grabec, S. Mandelj, Phys. Lett. A 287, 105 (2001).

[6] W.X. Ding, H. Deutsch, C. Wilke, A. Dinklage, Phys. Rev. E 55, 3769 (1997).

[7] T. Fukuyama, R. Kozakov, H. Testrich, C. Wilke, Phys. Rev. Lett. 96, 024101 (2006).

[8] V.I. Kolobov, J. Phys. D - Appl. Phys. 39, R487 (2006).

[9] B. Bruhn, B.P. Koch, C. Wilke, Contrib. Plasma Phys. 45, 328 (2005).

[10] M.A. Liebermann, A.J. Lichtenberg, Principles of plasma discharges and materials processing, (J. Wiley \& Sons, New York, 1994).

[11] H. Achterberg, J. Michel, Ann. Phys. (Leipzig) 2, 365 (1959).

[12] A. Garscadden, Ionization Waves in Glow Discharges, in Gaseous Electronics Volume 1, M.N. Hirsch and H.J. Oskam eds. (Academic, New York, 1978).

[13] K. Ohe, Current Topics Phys. Fluids 1, 319 (1994). 
[14] C. Wilke, B.P. Koch, B. Bruhn, Phys. Plasmas 12, 033501 (2005).

[15] S. Pfau, A. Rutscher, K. Wojaczek, Beitr. Plasmaphys. 9, 333 (1969).

[16] R.J. Deissler, Physica D 25, 233 (1987).

[17] A. Dinklage, C. Wilke, Phys. Lett. A 277, 331 (2000).

[18] A. Dinklage, B. Bruhn, H. Deutsch, P. Jonas, B.-P. Koch, C. Wilke, Phys. Plasmas 7, 883 (1998).

[19] M. Koepke, A. Dinklage, T. Klinger, C. Wilke, Phys. Plasmas 8, 1432 (2001).

[20] P.Jonas, B. Bruhn, B.P. Koch, A.Dinklage, Phys. Plasmas 7, 729 (2000).

[21] B. Bruhn and B.P. Koch, Phys. Rev. E 61, 3078 (2000).

[22] B. Bruhn, B.P. Koch and N.Goepp, Physica D 115, 353 (1998).

[23] Y.A.Kusnetsov, Elements of Applied Bifurcation Theory, Springer, Berlin, 1995, p. 312. 